\title{
The pedagogy of bio-design: methodology development
}

\author{
T. Roshko \\ Department of Interior Design, University of Manitoba, Canada
}

\begin{abstract}
This paper discusses the fundamental pedagogical objectives and methodologies that were employed to investigate the potential of bio-design as a valid platform for design education. The broader goal was to expand the boundaries of applicability of interior design and to encourage multi-dimensional design strategies in which sustainable design principles are embedded explicitly into the design process. Sustainable design can be defined as a cultural construct that enriches both environmental and social conditions with the purpose of nurturing the quality of life indefinitely at every level. In concert, bio-design is an interdisciplinary field, where man-made and natural systems intersect, and it has been exploited as a pedagogical platform in a graduate level design studio. The interior design students were allowed to engage in the iterative nature of biodesign methodology. As such, the investigation was engaged with the notion of "Nature as Culture" and aimed to re-establish the intrinsic human connection with Nature. This paper discusses the methodological development of bio-design studies in the context of Interior Design education.
\end{abstract}

Keywords: bio-mimicry, bio-design, bio-design education, sustainability.

\section{Introduction}

Man-made design and natural design are fundamentally different, and understanding this difference is the key to sustainable design. Man-made design is achieved through rational and, to some extent, intuitive design methodologies. On the other hand, biological or natural systems develop through evolution, in contrast to the rational methodologies of man-made systems. Nature's use of trial and error and the development of evolutionary solutions can act as a valuable resource and can inspire the design process. Bio-design work opens up a broad 
range of possibilities and sharpens the understanding of totalities and details. It provides a new method and perspective in the realm of creative work. Bio-design has not yet come of age for the principal reason that, from an engineering standpoint, Nature is quite complex. Evolution does not design by working towards a final goal, as would an engineer or a designer. Nonetheless, the gap with Nature is gradually closing; scientists are probing into the complexities of Nature with their electron and atomic force microscopes, micro-tomography and complex computer technologies. Bio-design is maturing into a commercial industry. Consequently, its inclusion into the design curriculum is quite timely.

The successful bio-design applications which have passed the conceptual or production stage are mostly in the field of materials development or product design. There is also a growing body of research which identifies obstacles to the implementation of bio-design as a viable design methodology in the field of architecture and interiors $[1,2]$. There is a clear lack of methodological approach for built environments in the field of bio-design, resulting in a limited number of field applications. Several approaches with varying degrees of sustainability outcome have been identified by various authors [1-4]. More often than not, biodesign is used either to produce novel ideas or to increase the sustainability of an existing design.

According to the literature, biomimicry as a design process can be summarized in two basic segments: design looking to biology for answers or biology looking to design [2]. The first approach, identified as the direct approach, requires a designer to formulate a design problem and a biologist to find a related design solution in nature. In this case, the initial objectives of the design are controlled by the designer; however, if the designer is performing the scientific research without an in-depth understanding of the topic, the translation of biological knowledge into human design has the potential to remain surface oriented. The second bio-design approach, which is labeled as the indirect method, is informed by Nature. Biological knowledge holds the biologist or ecologist responsible for the identification of a biological feature and its potential applicability to human design. The disadvantage of this method from a designer's point of view is that the designer has no control over the design parameters from the beginning of the process. This method has the greatest potential to contribute to human design in unprecedented ways, although it requires strong cross-disciplinary collaborations.

These methods can be applied at two different levels, the organism level and the ecosystem level. The application of bio-design methodologies at an organism level has the potential to neglect the contextual dependency of the organism and tends to limit the application to a specific part of the system rather than the whole system, thereby limiting the level of sustainability of the proposed design. Ecosystem level mimicry provides us with a design that goes beyond sustainability towards a system that is restorative [5]. In this study, the primary objective is to modify previously identified bio-design methods and to identify an emerging method which engenders a connection with Nature, fosters team and cross-disciplinary work and expands the possible boundaries of interior design. This study proposes that a sustainable design pedagogy must incorporate 
bio-design as one of its fundamental building blocks. The following sections discuss the background development and objectives of the design field in order to summarize the contextual definitions and theoretical framework of the study.

\subsection{Sustainability and design}

Our search for comfort, protection and convenience, and our desire to accumulate material wealth, has resulted in the breakdown of the natural systems which sustain us. Since the 1990s, the word sustainability has become one of the most commonly used words in our vocabulary, with a multitude of definitions. As an ideology, sustainability motivates us to connect with Nature, culture, politics, science and technology. It is perceived as a notion that evolved along two distinct streams. David Orr has labelled the two approaches as technological and ecological sustainability [6]. Technological sustainability seeks answers in technology and market solutions. Furthermore, the general belief is that we need experts to solve the environmental crisis. The overarching goal of the movement is to achieve a unified global management of sustainability, which has the great advantage of fitting within the current socio-political system. On the other hand, ecological sustainability finds its meaning in grassroots movements and local cultures and advocates the rethinking of our practices in agriculture, architecture, urban planning, transportation, resource management and all other core value systems. It has a natural aversion to our material wants, technology and most importantly our hubris. Currently, the Tri-Polar model, also known as Profit/Planet/People (3P), or Economics/Environment/Equity (3E), is used as a fundamental framework for sustainability. This current model is instrumental in combining the two streams of the sustainability movement. However, beyond acting as a mere model application, sustainability should be tightly woven into our lives at every level. The role of education in this process is undeniable and it is one of the primary focuses of this study.

David Orr has summarized the central reasons for the current sustainability crisis in five segments: the consequences of economic growth, social traps, our urge to dominate nature, the human condition and evolutionary development [6]. Other constructs from religion to colonialism to family structure have also contributed to lack of sustainability. However, Van Der Ryn and Cowan have succinctly argued that our environmental crisis is a design crisis [7]. They have articulated that it is a consequence of how we have made things, and constructed buildings, landscapes and cities. Our epistemology of design on which the current form of agriculture, engineering, architecture and industry is founded, is not compatible with that of Nature [7].

Van Der Ryn and Cowan have defined design as the intentional shaping of matter, energy and process to meet a perceived need or desire [7]. They claim that design is the connection piece which bridges culture to Nature through the use of materials, energy, and land use. It is implied that architects, city planners, interior designers, landscape architects and engineers along with farmers are all involved in shaping our physical world and are all designers [7]. So far we have used design to meet our exclusively defined needs without a careful consideration of the effects on other biological systems. 
Twentieth century architecture design was predominantly influenced by the metaphor of "building as a machine", where Nature was perceived as a passive backdrop. These static living machines did not allow exchanges or interactions with the larger context, Nature. In order to create a dialog between design and Nature our design epistemology requires a fundamental change. Not only our design praxis but also our design pedagogy is still based on $19^{\text {th }}$ century Beconian principles within which the notion of "domination over nature" constitutes the foundational thought process. A syllogistic approach to design problem solving and one-dimensional reasoning has resulted in non-interactive, static buildings and interiors which are driven by contracts and market economy. Furthermore, the users are perceived as mere customers. This is where the sense of place and local identities are lost. Global sameness has become pervasive; as a result, loss of regional differences undermines the uniqueness of place. Art and aesthetics are diminished and replaced with engineering and technology so that we can create our living machines more effectively. Furthermore, design is evaluated based on marketplace values, and is dependent on materials advancement, technological innovations and trends. In order to respond to the current disconnect between architectural design and Nature, we need to reevaluate our design pedagogy through the filter of sustainability.

\subsection{Linage of design pedagogy}

In response to the "new world" which appeared during the 1920s, Walter Gropius utilized a model which prioritized art and technology in the curriculum development of the Bauhaus School of Design in Weimar Germany. Science was the underprivileged segment of the trinity in the art, science and technology based curriculum. By contrast, the model for the New Bauhaus School of Design in Chicago, developed during the 1930s by Moholy-Nagy, was based on art and science. Tomas Moldanado once again celebrated the coupling between technology and science to inform his curriculum for the Hochschule Fur Gestaltung, in New Ulm during the 1950s in response to the "new culture" which embraced science and technology as the ideal [8]. Today we are still looking at the Bauhaus lineage to inform our epistemology of design. While it is undeniable that art, science and technology are all part of the archetypal tri-polar model, there are nevertheless great variations in the level of emphasis placed on the individual components.

Allan Findeli argues that in order to find coherence in the curriculum we must define the overall purpose of design education and practice [8]. Currently, we are preoccupied with a culture-based approach to design. Findeli invites us to consider a new theoretical model whose theoretical framework is inspired by system logic, complexity theories, and practical philosophy [8]. In his vision, visual intelligence and technology as a moral act replace the science and technology component of the model. Finally, he modifies the art component with a phenomenology based aesthetic intuition [8].

Findeli defines visual intelligence as an ability to see the world in its complexity and interrelatedness. He defines the interrelated subsystems which operate on different logic as the man-made world, the biophysical world, the 
social world and the symbolic world [8]. He argues that design projects operate within the complex assembly of these inner and outer worlds. Therefore, the visual intelligence of the designer must be cable of delving into these different realms with dexterity. He reminds us of Moholy-Nagy's words, "design is not a profession but an attitude" [8]. The designer, as a synthesizer of the emerging realities, must alter his or her attitude to reflect our ecological responsibilities with renewed visual intelligence.

During the industrial revolution, design acted as the mediator to soften the effects of industrialization on the socio-cultural realm using aesthetics as a tool. Later, modern and post-modern society demanded that design embrace, as mediator, ergonomics and semiotics respectively [8]. Today we are looking at design once again as a medium to soften the consequences of accelerated science and technology. We want our built environments to be socially, culturally and economically viable [8]. The role of designers is shifting from service industry worker to interpreter of the new complexities of our living world. Designers are asked to recognize the complexities of the world and to offer simplicity in their solutions. In order to fulfill the role of a synthesizer of knowledge, designers have a renewed purpose in their design education. Although the general purpose of design has developed within the Bauhaus tradition, the new purpose of design must be firmly based on the central concerns of the biological world. In addition, socio-cultural and economic sustainability should be considered alongside with ecological concerns. Therefore, technological sensitivity becomes an issue of ethics. Designers are part of the eco-system and should be armed with knowledge of ecological and technological ethics.

The undisputed role of the creative aspects of design has been acknowledged by current pedagogical models; however, our age demands to see everything in relationship and demands the dexterity to scale-link across the board from product to urban design. Although the system of science can be perceived quantitatively, other systems such as human and social systems are best understood from a qualitative, phenomenological point of view. The phenomenology of human and social systems demands an existential point of view and must respect the intuitive process of aesthetic development.

Sustainable design can also be defined as a cultural construct that enriches both environmental and social conditions with the purpose of nurturing the quality of life indefinitely at every level. The primary motivation of the discipline of Interior Design is to answer both the emotional and physical needs of human beings. North American culture spends close to $85 \%$ of its time indoors and this has had a profound effect on sustainable design developments and, consequently, on the quality of life [9]. Interior Design is thus positioned at the centre of the sustainability paradigm. In spite of this, it is very difficult to define human well being, since it is culturally related, context specific, cannot be prioritized and has a strong dependence on time. By considering the universal motivational forces such as physical survival, communicating with others, creating things and having a sense of self, Ann Thorpe has defined the quality of life in terms of nine interrelated areas: subsistence, protection, affection, understanding, participation, leisure, creation, identity and freedom [10]. Thorpe 
maintains that human well-being can be achieved when these needs are constructively satisfied [10]. These needs are considered to be emotional and intellectual in nature and are directly linked with interior design practice and education.

The collaboration between designers and scientists has produced tangible results in the advancement of the well-being of our society in the fields of healthcare, climate change and energy conservation. Team work and the multidisciplinary nature of design must be an integral part of design studies at the pedagogical level so that the structure of sustainability, the connection between sustainability and innovation and, as a result, the emotional engagement with sustainability can be understood.

The graduate level Interior Design Materials studio, within which the bio-design studies were housed, was designed with the objective of addressing the sustainability of human well-being, for which the designer plays a critical role as the key provider of images, objects and interior spaces. The learning objectives, which are founded on the Findeli model, can be listed as follows: renew our connection with nature, develop technological ethics, develop and acknowledge the intuitive development of design aesthetics, foster a notion of teamwork, develop methods to see the world in its various complexities, learn to scale-link, and finally develop scientific methodologies to expand the interior design operational and methodological boundaries.

\section{Bio-design}

Nature as culture is not a new concept and has been expressed at every level of the design process, including as a form and aesthetic determinant, from early human history. Nature has inspired artists, architects and engineers throughout the centuries. Natural shapes, patterns, structures, construction principles and techniques have been utilized in many ways and forms. There are countless illustrations of this in the works of artists and architects, from Leonardo da Vinci to Callatrava.

The definition of bio-design evolved and was refined through the 1980s and, at the turn of this century, it was defined by Meyer's Grosse Taschenbuchlexikon in Manheim as follows:

"The interdisciplinary field of Bio-design encompasses systematic studies of functions, relations, structures and processes in biological systems and transforms these data into solutions of primarily technical and technological problems..." [11]

The field was summarized succinctly and effectively by the American pioneer of bio-design studies, Janine Benyus, as:

"Nature as model, measure and mentor" [12]

Students were asked to consider the basic principles of nature, as articulated by Benyus [12]. 


\subsection{Bio-design course structure and methodology}

The bio-design portion of the studio was positioned at the merger of Interior Design with Biological Science and Scientific Methodologies with the purpose of developing the notions of the ethical application of technology and visual intelligence. The essence of bio-design lies in understanding the basic principles of design in Nature. Bio-design can begin with a well-defined problem, where solutions can be reached through the analysis of a natural system. Subsequently, the fundamental principles of natural systems are abstracted and eventually translated into a new design form.

Prior to project initiation, loose boundaries were established in order to provide a framework for problem development. The areas of interest were selected to be the design of an enclosure, a partition or an engagement with the concept of illumination at any scale, with each utilizing bio-design principles within the context of interior design or product design. Three different biodesign methods of problem solving were introduced sequentially, starting with the direct approach, followed by the indirect approach and finally the hybrid approach.

\subsubsection{Direct approach}

Students were asked to define a specific design problem within the loosely identified design areas. Following the identification and articulation of the design problem, the direct approach strategy was divided into three distinct stages: Analysis, Translation and Implementation. Students were asked to search for an organism or ecosystem which addressed a similar problem. The analysis stage was further developed through the exploration of existing biomimicry databases and published literature. Students were encouraged to examine biological systems on all scales, from the single organism level to the ecosystem level, in terms of their form, function, structure, material and behaviour in order to develop a basic understanding of the governing principles. These principles were subsequently simplified, abstracted and then applied to generate a solution. The analysis and translation processes were fairly straightforward once the facts and general principles of the biological system were understood. The project duration was limited to one week and, as a result, the proposals were limited in scope. A drawback of the process was the tendency to over-simplify the natural principles. However, this stage was viewed as foundational and orientational, with the primary purpose of familiarizing the students with bio-design methodologies and techniques.

SEED-LET was a proposal by Andrea Ewanchyna, Jan Hallick and Vanessa Ilg which resulted from the investigation of the magnolia seed pod. Their initial design problem was to create an enclosure which would recognize and soften the period of transition between the mother's womb and the child's entry into the world. Plug-in simulators were designed to emulate the mother's scent and heart beat, while material investigations resulted in jell-tile inserts, which created additional support, as well as a continuation of the buoyancy of the placenta. The team proposal for the baby-carrying case also included two wearing options, one 

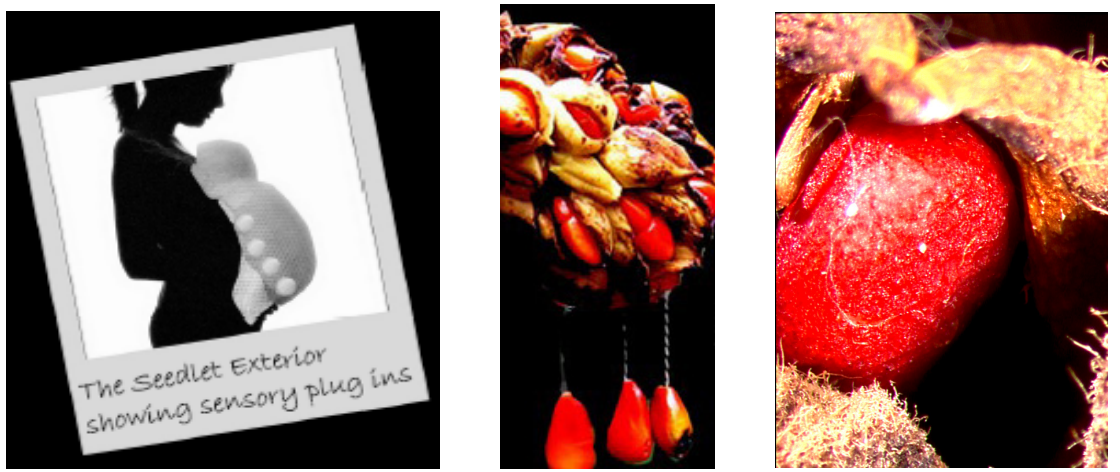

Figure 1: $\quad$ Seed-Let Volume Studies; encapsulation process of a magnolia seed pod informed the form development. Similarly, the structure of the connection of the seeds to a pod inspired the plug-in simulators.

which could be used close to the mother's body, and the other in a hanging position away from the mother's body, with the plug-in simulators activated. See Figure 1.

\subsubsection{Indirect approach}

The students were first asked to select natural objects from an extensive list at the early stages of the object Analysis process, without any specific problem in mind. These included fruits, leaves, vegetables, flowers, seeds and seed pods, bones and skulls, integuments (snake and turtle), mammal horns/claws/hooves, feathers, glass sponge, corals, shellfish (Nautilus, snails, clams, scaffopods), sea urchins, starfish, various insects, silk moth cocoons and butterfly pupae and wings. The area of investigation was limited to the three predetermined areas of design as previously mentioned. Nevertheless, this new role of "designer as biologist" overwhelmed the students at first. Professor Erwin Huebner from the Department of Biological Sciences in the Faculty of Science was invited to participate in the role of visiting expert. His research laboratory, and specifically his dissecting microscope, complete with optical network, was used as the primary investigation and data collection centre. Students were tutored by Dr. Huebner in data collection and image capture at the micro-level with the dissecting microscope. His participation brought clarity to the investigations and alleviated the initial reservations of the students. The duration of the project was limited to four weeks, and Dr. Huebner's considerable input at every level helped to foster an appreciation for a cross-disciplinary approach to problem solving, and aided students in the development of their scientific skill set.

The students were asked to re-examine the Nature-based design principles that were deduced at the end of the Analysis stage, and to simplify them if necessary in order to understand and clarify the basic principles of form, function, structure, and materials. During this Analysis stage, students started to establish the framework of a design problem which is derived or, more appropriately, inspired directly from the biological material. An iterative process 
of bio-design investigation led the students to solidify the design problem. Eventually the students were able to translate Nature-based principles of form, function, structure, material and behaviour into a design proposal. The final Implementation stage required the students to modify and re-evaluate the biodesign based solution and to initiate the final implementation of the design. The final model was expected to represent the relationships between Form-Function, Form-Structure, Whole-Parts, and Construction-Material. The indirect approach inspired more creative solutions and novel ideas than the direct approach. This form of investigation provided ample opportunities for students to see Nature as a source of design inspiration and to understand its infinite complexities within a design context. By developing their range of visual intelligence and cultivating a close engagement with Nature, the students were given the opportunity to base their notion of design aesthetic on Nature rather than on trends.

The bio-design investigations of the team of Amber Bewza, Naomi Dudridge, Anna Westlund, and Min Young Kim resulted in a prototype proposal for C-FLO. C-FLO is a personal transportation device (PTD) whose design was inspired by the form, function and structure of the nautilus. The name was derived from the class of nautilus known as Cephalopda. The team was inspired by the basic principle of mobility of the nautilus. When sea water enters the mantel cavity of the nautilus, it causes the radial neck muscles to contract and to form a tight seal around the neck. As a consequence, the high speed expulsion of water from the mantel cavity of the Cephalopda provides propulsion and long range mobility to the nautilus. The mobility of C-FLO was based on a similar principle, wherein a foot pump was located at the floor level of the PTD to simulate the propulsion effect. The prototype was designed for recreational use. See Figures 2 and 3.

Michelle Zelickson and Hailey Connor were fascinated by the life cycle of a wild cumber and centred their investigation on both the micro level and macro level properties of the plant. After the preliminary investigation, they extracted a list of characteristics and associated these characteristics with a possible set of human design problems. The properties of the wild cucumber seed pod such as its ovoid form, its hydraulic system of seed ejection, its multi layered and light weight structure and its ability to use space and structure efficiently led the group

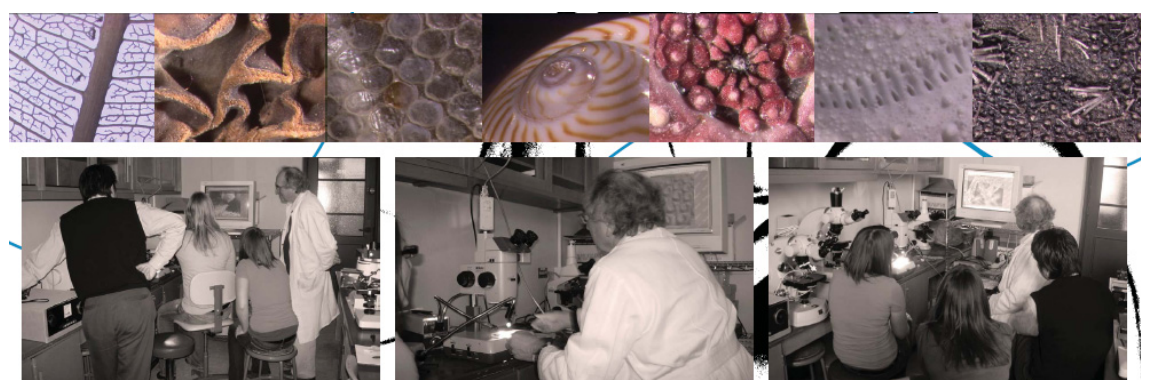

Figure 2: C-FLO laboratory observation. 


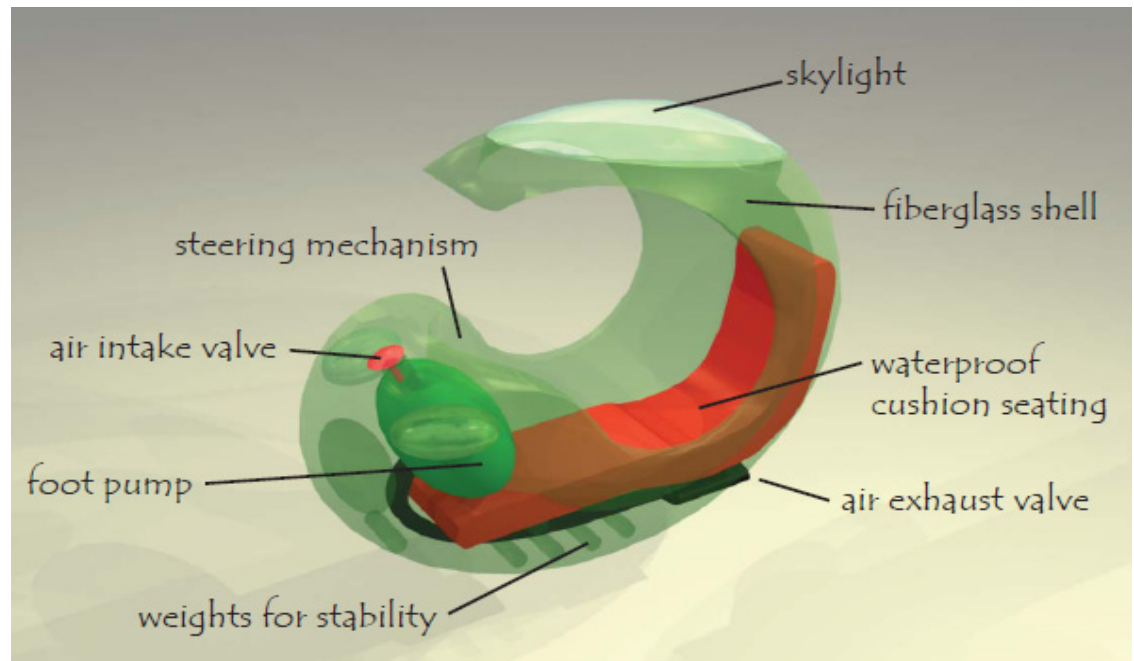

Figure 3: $\quad$ C-FLO design development proposal.
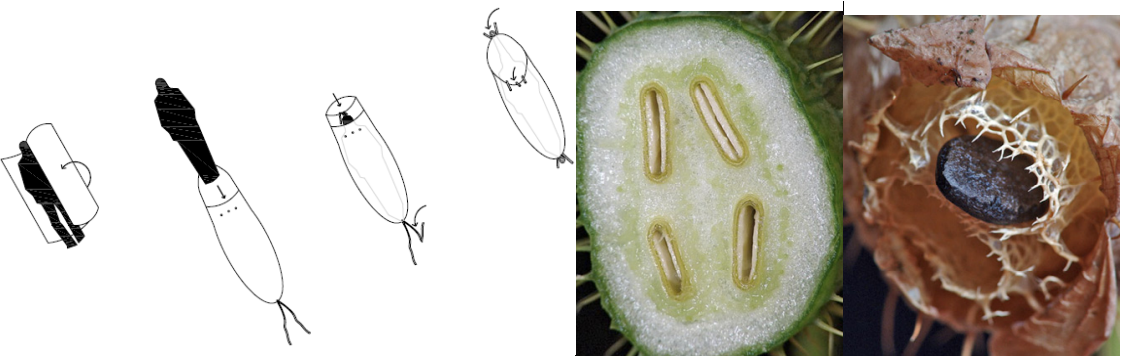

Figure 4: Return to Earth Casket (R-TEC) inspired by wild cucumbers. An investigation into the burial rituals of the major religious groups resulted in Return to Earth Casket (R-TEC) which took the form of an ovoid shell with multi layers. In the proposal, a linen or cotton shroud is followed sequentially by loosely and then tightly woven hemp shells.

to a proposal for an environmentally conscious casket. The group rationalized the process as follows: "The structure, form and function of the R-TEC are closely linked to one another. The form and spacing of the layers, as well as the materiality of the casket, are integral to assisting the biodegradable processes and have a wide range of applicability to various burial rituals". See Figure 4.

\subsubsection{Hybrid approach}

Even though direct and indirect approaches were clearly identified as distinct modes of study in the course brief, some of the students used a hybrid approach 
to their bio-design work. They started with a very well defined problem and then proceeded with the analysis process of a biological object which holds the solution to a similar design problem in nature. The investigation of the biological object, and the discovery of new facts, deeply inspired the students to reformulate the original design question. Weaving between "design looking into nature" and "nature informing design" modes further inspired students to integrate interior design theories into their study. As a consequence, their proposals displayed a greater range of creativity, interdisciplinarity and novelty of ideas. At the end of each segment, students were required to verify their solutions against the "life" criteria listed by Janine Benyus [12] to ensure the integrity of the proposed bio-design based solution independent of the approach. Once the answer to the "life" criteria is "yes", one can claim that the proposed design is bio-design based and has precedent in Nature. Furthermore, students were asked to consider the following rule: transfer of form from Nature for aesthetic purposes alone, regardless of its appeal, will not be considered a valid bio-design based proposal.

Urban Privacy Pod was generated at this hybrid intersection. It was the result of studies done by Ashley Jull, Pricilla Mah, Janine Shwaluk and Kristie Spencer. This group initially wanted to design an efficient packaging system and started by investigating a series of biological objects. The Venus flower basket was one of the objects of choice; however, an intense analysis stage revealed that the sea sponges usually house and/or trap a pair of shrimp for the duration of their life span. This greatly affected the group and caused a change in direction. They rearticulated the design problem as a possible design for a personal enclosure that could be used within the public realm. The bio-structure of the Venus flower basket was used as the design inspiration. After intense microscopic and macroscopic structural studies of the glass sponge, the team was inspired by its structural complexities and crystalline double helix configuration, which ultimately formed the basis for the proposed privacy pod. The light transmitting fiber optic properties of the anchoring strands, and the parasitic qualities of the sponge, also informed the design and the contextual application

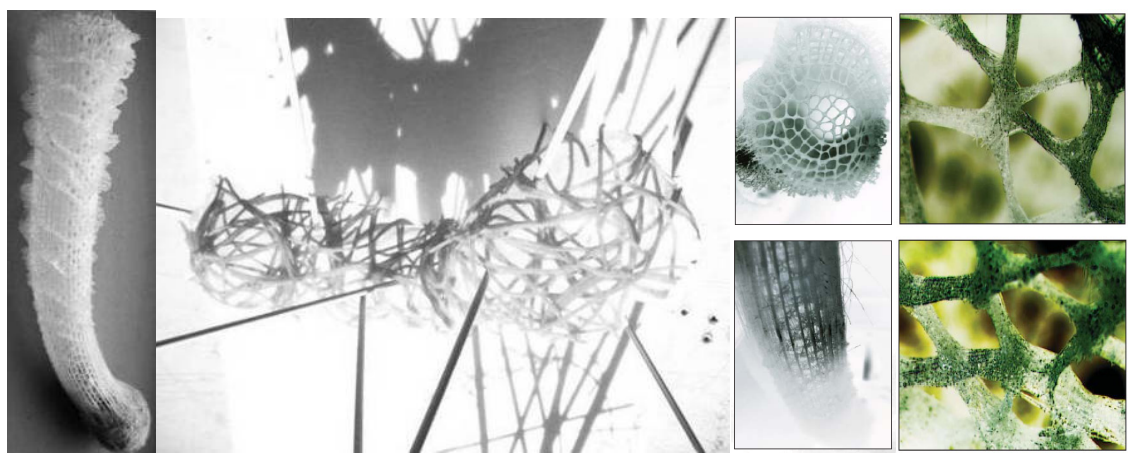

Figure 5: Venus flower basket; form, structure and material studies allowed design students to develop the confidence to weave between the roles of designer, biologist and engineer. 


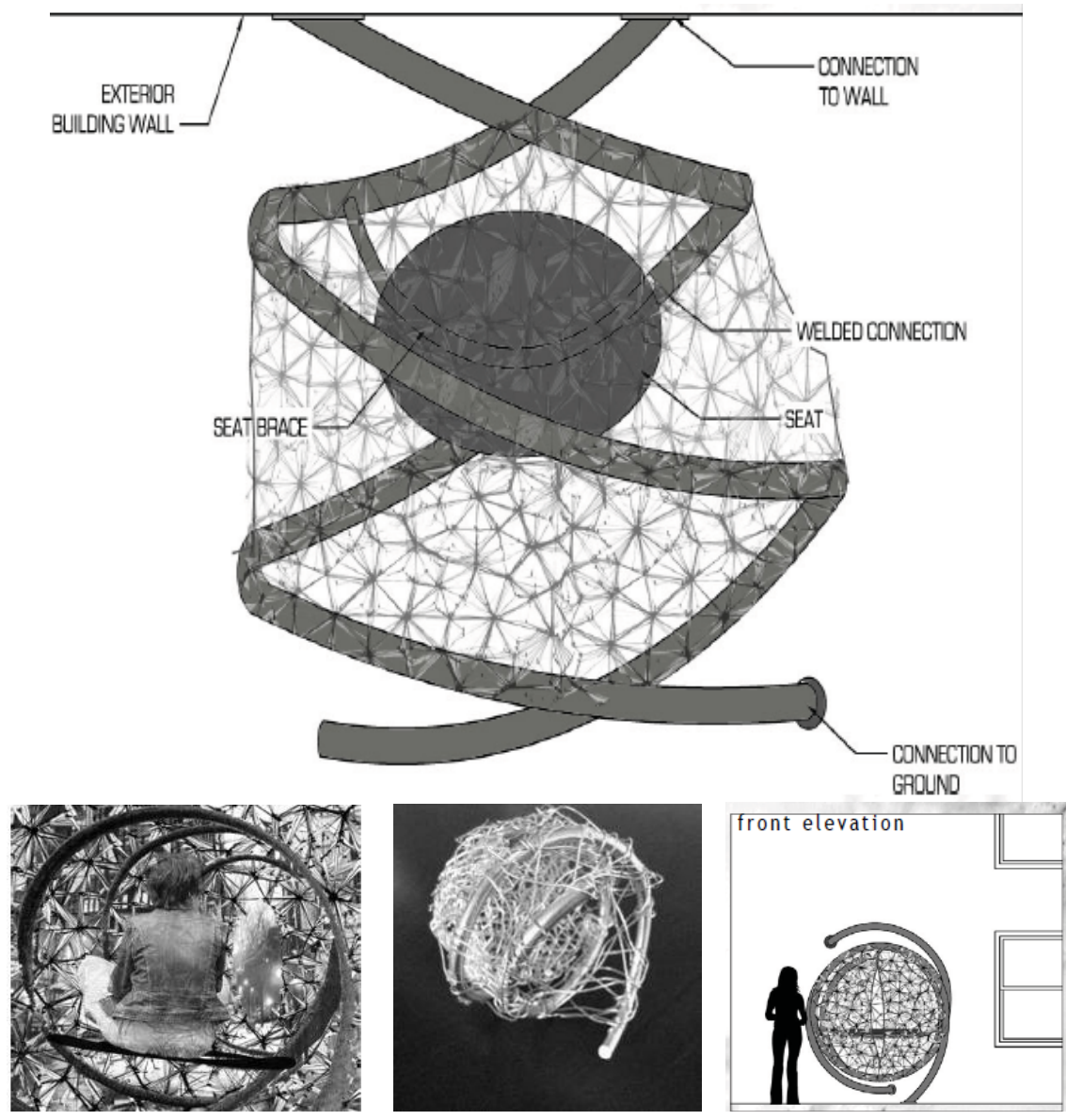

Figure 6: Urban Privacy Pod design development, plan, volume and elevation.

development of the proposal. The team defined the "third place" as a point of anchor of community life, which fosters a broader, more creative interaction. Their urban privacy pod was perceived as the "fourth place", where the semiprivate functions can take place within the public realm. See Figures 5 and 6.

\section{Concluding remarks}

Bio-design pedagogical methodologies were modified during the course of three different bio-design studios. The Hybrid type approach had the greatest potential. In this approach, the design problem formulation is shifted from the initial stages to the end of the analysis stage, thus allowing the designers to exercise their creative ability and to synchronize it with that of Nature. Rather than a tightly structured methodological approach, a combination of formal approaches allowed designers the freedom to resume the role of a designer with a renewed 
sense of appreciation of the scientist's contribution. The Hybrid approach also allowed the designers to use biological form as a source of inspiration at any stage, as long as it is supported by function and structure studies. Findeli's approach to a new design curriculum was used as the foundation for establishing a viable bio-design methodology in the studio. The emerging hybrid method does not privilege either the designer or the biologist as the driving force behind the design process, but instead is built around an equal collaboration. This method has more possibilities for interior designers, even though the biomimicry is performed at the organism level. The evolution of bio-design methodologies is similar to that of Nature. Trial and error will result in new morphologies and solutions. Perhaps built-in redundancies in the process will prepare the emerging bio-design education to combat the vacillating changes in the ecology of design.

The role of designer as biologist created quite a high level of anxiety in the class during the execution of the direct methodology segment, and the participation of a professional biologist was essential to the success of the project. Otherwise, the students exhibited a strong inclination to mimic form and some basic structural elements only. Regardless of the difficulties associated with the direct approach [1,2,4], it offers viable contributions to the sustainability level of a building. It has been argued that the incremental improvement of current un-sustainability in our buildings is not the answer. While our design thinking is undeniably in need of change at a fundamental level, it is nevertheless quite feasible to exploit the cumulative effects of concerted efforts in order to improve sustainability. An example is the building of a Chimera as promoted by Janine Benyus [13]. Although the indirect methodology extended the project duration due to the extensive biological research, it yielded more complex solutions and gave the designers the opportunity to share the control of the design process with scientists.

As science and technology continue to accelerate and change our society, design has become the primary mediator in the implementation of these changes in our daily lives. A new breed of intellectuals, who have the ability to grasp the changes in science and to convert them into objects or ideas which can be implemented, must be fortified with knowledge of technological and ecological ethics. Due to the lack of expertise on the topic of "ecological and technological design ethics" in the studio, it was rather difficult to establish ethical boundaries which were limited to and defined by individual moralities and values. Furthermore, the bio-design work was mostly performed at the organism level; future bio-design studios which focus on ecosystem level mimicry will have greater potential, since the correlations between natural and urban ecologies are quite substantial. The future development of bio-design methodologies in the studio would also benefit from the inclusion of a structural engineer and an ecologist to complete the future model of a design/education team which is armed with an inherent knowledge of ecological and technological ethics. 


\section{Acknowledgements}

Amber Bewza, Naomi Dudridge, Andrea Ewanchyna, Jan Hallick, Vanessa Ilg, Ashley Jull, Min Young Kim, Pricilla Mah, Janine Shwaluk, Kristie Spencer, Anna Westlund. All images are produced by MID Class of 2008, except figure 4. Figure 4 is prepared by Michelle Zelickson, Hailey Connor, in MID Class of 2009. Special thanks to Dr. Erwin Huebner for his assistance during the Bio-design segment of the studio.

\section{References}

[1] Faludi, Jeremy, Biomimicry for Green Design (A How-To), 2005, accessed on June 25, 2009, www.worldchanging.com/archives/003680.html

[2] Pederson Zari, M., Biomimetic Approaches to Architectural Design for Increased Sustainability, paper no: 033, presented at (SB07) Regional Sustainable Building Conference, Auckland, New Zealand, Nov 2007.

[3] Biomimicry Institute official web site, accessed on November 5, 2009 www.biomimcryistitude.org/about-us/biomimicry-a-tool-forinnovation.html

[4] Kepler, J., Stokholm M., Bionic Design Methods-A Practical Approach, Proceedings of the 4th international Conference on Advanced Engineering Design, AED 2004; Glasgow, Scotland, UK; Sept. 5-8, 2004.

[5] Kellert, S., Heerwagen, J., H., Mador, M., (Eds.), Biophilic Design, New Jersey: John Wiley and Sons, pp. 5, 135, 2008.

[6] Orr, D., Ecological Literacy: Education and the Transition to a Post Modern World, Albany: State University of New York Press, pp. 24; pp. 4. 1992.

[7] Van Der Ryn, S., Cowan, S., Ecological Design:10 ${ }^{\text {th }}$ Anniversary Edition, Washington, Covelo, London: Island Press, pp. 24; , pp. 24, 168. 2007

[8] Findeli, Alain, Rethinking Design Education for the $21^{\text {st }}$ Century: Theoretical, Methodological, and Ethical Discussion, Design Issues, 17(1). pp. 5-17; pp. 13; pp. 16; pp. 10-16; pp. 11; pp. 15, Winter 2001.

[9] Bernardi, J. \& Harmon-Vaughn, B., Teaching Interior Design StudioBased on a Collaborative Process, Social Embeddedness and Sustainable Design, Thinking inside the box: a reader in interiors for the $21^{\text {st }}$ Century, eds. J. Gigli, Hay, F., Hollis, E., Milligan, A., Milton, A., Plunket, D, London: Middlesex University Press, pp. 253-258, 2007.

[10] Thorpe, Ann., The Designer's Atlas of Sustainability, Washington, DC: Island Press, pp. 114-115, 2007.

[11] Bionics in Meyers Grosse Taschenbuchlexikon, Mannheim Bibliographishes Institute, 1983.

[12] Benyus, Janine. Biomimicry: Innovation Inspired by Nature, New York: Harper Collins Publishers Inc., pp. xi.; pp. 7, 291-292, 1997.

[13] Benyus., J., A Good Place to Settle: Biomimicry, Biophilia, and the Return of Nature's Inspiration to Architecture (Chapter 3), Biophilic Design, eds. Kellert, S., Heerwagen, J., H., Mador, M., New Jersey: John Wiley and Sons, pp. 30, 2008. 